\title{
Adaptación del modelo canvas como herramienta competitiva para las mpymes de Lázaro Cárdenas, Michoacán*
}

\author{
Adaptation of the canvas model as a competitive tool for the \\ mpymes of Lázaro Cárdenas, Michoacán.
}

\section{Adaptação do modelo de lona como ferramenta competitiva para os mpymes de Lázaro Cárdenas, Michoacán.}

\author{
Rafael Casas-Cárdenas \\ Instituto Tecnológico de Lázaro Cárdenas, Lázaro Cárdenas - México \\ cacrcpc@hotmail.com \\ José Vargas-Hernández \\ Universidad de Guadalajara, CUCEA, México \\ josevargas@cucea.udg.mx \\ Rebeca Almanza-Jiménez \\ Instituto Tecnológico de Lázaro Cárdenas, Lázaro Cárdenas - México \\ rebeca_aj@hotmail.com
}

F. Recepción: 05 mayo 2017

F. Aceptación: 20 junio 2017

\begin{abstract}
Resumen
Las micro, pequeñas y medianas empresas representan un papel importante en la economía de México, principalmente en la generación de empleos y su contribución en el producto interno bruto. El Plan Nacional de Desarrollo 2012- 2018, las considera como una palanca estratégica para el desarrollo nacional y la generación de bienestar. Sin embargo, los apoyos gubernamentales no han sido suficientes, en parte por los efectos de la reciente crisis económica global. Por lo anterior es necesario que se busquen diferentes estrategias que contribuyan en su desarrollo, evitando su estancamiento y en muchos casos la desaparición. Como respuesta a esta problemática nace la presente investigación con la finalidad de contribuir con estas unidades económicas mediante la adaptación del modelo de negocios canvas como una herramienta que contribuya en su competitividad.
\end{abstract}

* Cómo citar: Casas-Cárdenaz, R., Vargas-Hernández, J.V., Almanza-Jiménez, R. (2017). Adaptación del modelo canvas como herramienta competitiva para las mpymes de Lázaro Cárdenas, Michoacán. Revista Libre Empresa, 14(2), 83-103 http://dx.doi.org/10.18041/libemp.2017.v14n2.28205 


\title{
Palabras clave
}

Competitividad, modelo canvas, mpymes, plan de negocios

\begin{abstract}
Micro, small and medium enterprises represent an important role in the Mexican economy, mainly in the generation of jobs and their contribution to the gross domestic product. The national development plan 2012-2018 sees them as a strategic lever for national development and the generation of well-being. However, government support has not been sufficient, partly because of the effects of the recent global economic crisis. Therefore, it is necessary to look for different strategies that contribute to its development, avoiding its stagnation and in many cases the disappearance. As a response to this problem, the present investigation was born in order to contribute with these economic units by adapting the canvas business model as a tool that contributes to its competitiveness.
\end{abstract}

\section{Keywords}

Competitiveness, canvas model, mpymes, business plan.

\section{Resumo}

As micro, pequenas e médias empresas representam um papel importante na economia mexicana, principalmente na geração de empregos e sua contribuição para o produto interno bruto. O plano de desenvolvimento nacional 2012-2018 vê-los como uma alavanca estratégica para o desenvolvimento nacional e a geração de bem-estar. No entanto, o apoio do governo não foi suficiente, em parte devido aos efeitos da recente crise econômica global. Portanto, é necessário procurar diferentes estratégias que contribuam para o seu desenvolvimento, evitando a sua estagnação e, em muitos casos, o desaparecimento. Como resposta a este problema, a presente investigação nasceu para contribuir com essas unidades econômicas, adaptando o modelo de negócios de tela como uma ferramenta que contribui para a sua competitividade.

\section{Palavras-chave}

Competitividade, modelo de lona, mpymes, plano de negócios.

\section{Introducción}

Las micro, pequeñas y medianas empresas, de conformidad con el INEGI, son de gran importancia en la economía y en el empleo nacional y regional, tanto en países industrializados como en los de menor grado de desarrollo. Actualmente la desaceleración económica global, incluyendo México, genera altos índices de desempleo, representando las micro, pequeñas y medianas empresas, una alternativa para generar nuevas fuentes de trabajo y contribuir al desarrollo económico en su área de influencia. 
Actualmente, ante la incertidumbre que vive el país derivada de la deportación masiva de inmigrantes y la renegociación del Tratado de Libre Comercio de América del Norte, las mpymes requieren, además de apoyos gubernamentales para su desarrollo, estrategias que contribuyan en su competitividad, generando nuevas entidades y el crecimiento de las ya existentes, para favorecer de esta manera los indicadores en empleabilidad.

El modelo canvas para generar un plan de negocios, es una herramienta de fácil manejo, diseñada para agregar valor y contribuir en el éxito de los negocios. Una de las principales características de las mpymes es que son empresas familiares y generalmente son dirigidas por uno o más de sus miembros, careciendo de una estructura formal y de los conocimientos necesarios que permitan establecer procesos que desarrollen su competitividad en un entorno cada vez más globalizado. Este modelo de aplicación sencilla, adaptado de manera precisa, puede convertirse en una alternativa para los emprendedores de nuevas empresas o las ya existentes, para visualizar y dar forma a un plan de negocios exitoso.

Sin embargo, los modelos de negocios son una respuesta en un tiempo y espacio determinados, atendiendo necesidades identificadas por el autor de los mismos, pero representan un instrumento básico para su adaptación en diferentes entornos y escenarios económicos, sociales y culturales. Es así como nace la idea de investigar y adaptar el método canvas para su aplicación en las mpymes de Lázaro Cárdenas, Michoacán, como una herramienta que contribuya a su competitividad.

\section{Antecedentes de las mpymes}

En México, en el diario oficial de la Federación del día 30 de diciembre de 2002, se publicó la ley para el desarrollo de la competitividad de la micro, pequeña y mediana empresa, teniendo como objetivo promover el desarrollo económico nacional a través del fomento a la creación de micros, pequeñas y medianas empresas y el apoyo para su viabilidad, productividad, competitividad y sustentabilidad. Asimismo, incrementar su participación en los mercados, en un marco de crecientes encadenamientos productivos que generen mayor valor agregado nacional. Esta ley sigue vigente, lo que hace evidente el interés por fortalecer su competitividad frente a la creciente globalización; sin embargo, los programas de apoyo han sido insuficientes, lo que se requiere en principio es conocer las características y necesidades de las mpymes para establecer las estrategias que contribuyan de manera eficiente en su competitividad.

Esta ley tiene como finalidad fomentar el empleo y el bienestar social y económico de todos los participantes en la micro, pequeña y mediana empresa. Uno de los retos prioritarios de las últimas administraciones en México, pues según los datos de INEGI el crecimiento de la economía ha estado del orden del 2.3 en el año 2016 y los años anteriores el comportamiento ha sido similar, lo que refleja un déficit comparado con el índice de inflación que durante dicho periodo fue del $3.36 \%$. 
A. De acuerdo con los datos del último censo presentados por INEGI, en la ciudad de Lázaro Cárdenas, Michoacán, existen 4.816 mpymes, distribuidas en las principales actividades estratégicas, como se muestra en la Tabla 1.

Tabla 1.

MPYMES en Lázaro Cárdenas, en actividades estratégicas.

\begin{tabular}{|l|c|}
\hline \multicolumn{1}{|c|}{ Actividad } & Número de establecimientos \\
\hline Construcción & 54 \\
\hline Industrias manufactureras & 731 \\
\hline Comercio al por mayor & 193 \\
\hline Comercio al por menor & 3.645 \\
\hline Transporte & 193 \\
\hline \multicolumn{1}{|c|}{ Total } & $\mathbf{4 . 8 1 6}$ \\
\hline
\end{tabular}

Fuente: Elaboración propia

Considerando los datos consignados en la Tabla anterior, las micro empresas o comercio al por menor representan una cifra de 3.645 unidades, lo que porcentualmente corresponde a un $76 \%$ del total de establecimientos considerados. Lo anterior justifica que este grupo de empresas sea impulsado para que se consoliden y crezcan, beneficiando el entorno económico de la región y del país, mediante apoyo gubernamental económico y administrativo, modelando herramientas de fácil aplicación que permitan impulsar su desarrollo y competitividad.

Uno de los grandes retos actualmente, lo representa la creación de empleo, dados los niveles de crecimiento de la economía en los últimos años en el país, así como el escenario adverso ante la política migratoria de Estados Unidos, todo esto obliga a buscar opciones de empleabilidad y crecimiento interno, lo que requiere aprovechar los recursos con que se cuenta. México es un país con una muy alta concentración de empresas de menor tamaño, en especial de las denominadas micro, las cuales son $95 \%$ del total de empresas y absorben $41.83 \%$ del total del personal ocupado. En términos agrupados, las mpymes generan $73 \%$ del empleo total (Góngora, 2013).

B. Características de las mpymes en México.

Para poder entender de manera más precisa la integración de este grupo de empresas, es necesario abordarlas de manera individual, como a continuación se describe.

\section{1) Microempresas}

Las microempresas, de acuerdo con la estratificación publicada en el diario oficial de la Federación el 30 de junio de 2009, considera aquellas con hasta diez trabajadores e ingresos hasta cuatro millones de pesos. Gran cantidad de estas empresas se encuentran 
en la informalidad por diversas razones, primeramente por falta de control por parte de los diferentes niveles gubernamentales, seguido de la falta de una verdadera simplificación en el pago de impuestos y cumplimiento de obligaciones de carácter fiscal. Aunado a lo anterior, su agrupamiento en organizaciones que son utilizadas para fines políticos. Todo ello contribuye a su rezago y su poca contribución en el crecimiento de la región y del país. En cuanto al empleo como se ha mencionado, juegan un papel importante, sin embargo dicha empleabilidad igualmente cae en la informalidad por falta de control oficial.

Se comenta que en cuanto al empleo, las microempresas caen en la informalidad, al no contar con un registro formal ante las autoridades federales, estatales y locales, lo que origina que este tipo de empresas no otorguen las prestaciones básicas, tales como seguridad social, vacaciones, prima vacacional y participación en las utilidades, de igual manera no contribuyen en el pago de impuestos. Durante el primer trimestre de 2016, 29.1 millones de personas conformaron el empleo informal, más de la mitad de la población ocupada, lo que representa un crecimiento de 1.5\% frente al mismo periodo de 2015, informó la Encuesta Nacional de Ocupación y Empleo (Forbes, 2016).

\section{2) Pequeñas empresas}

De acuerdo con la estratificación en México de las micro, pequeñas y medianas empresas, quedan comprendidas como pequeñas aquellas que emplean de 11 a 30 trabajadores, tratándose de actividades comerciales y de 11 a 50 empleos en actividades de industria y servicios; en cuanto a ingresos de 4 a 100 millones de pesos para los dos sectores. Este tipo de establecimientos por disposiciones fiscales, principalmente, por su nivel de ingresos y necesidades de control, así como por programas de verificación de la autoridad, se encuentran registradas ante el sistema de administración tributaria, de la misma manera para efectos del otorgamiento de las prestaciones de seguridad social, sin embargo, al igual que las microempresas, carecen de sistemas administrativos y financieros que les permita un crecimiento sostenido y el desarrollo en su competitividad.

\section{3) Medianas empresas}

En cuanto a medianas empresas, según la estratificación referida y que más adelante se menciona, están consideradas en el ramo de comercio aquellas que cuentan con 31 a 100 trabajadores, tratándose de servicios de 51 a 100 y del ramo industrial de 51 a 250. Con respecto a los ingresos de 100 a 250 millones de pesos anuales en las tres actividades descritas. Considerando las actividades estratégicas, según el último censo económico realizado por el Instituto Nacional de Estadística y Geografía, en 2009 se cuenta en la Ciudad de Lázaro Cárdenas Michoacán, con 75 establecimientos.

La estratificación de micro, pequeña y mediana empresa a que se hace referencia en los párrafos anteriores, entró en vigor mediante acuerdo publicado en el diario oficial de la Federación del 30 de junio de 2009, conforme a los criterios que en la misma se establecen. 
Tabla 2.

Estratificación de micro, pequeña y mediana empresa

\begin{tabular}{|c|c|c|c|c|}
\hline Tamaño & Señor & $\begin{array}{l}\text { Rango de } \\
\text { número de } \\
\text { trabajadores }\end{array}$ & $\begin{array}{l}\text { Rango de } \\
\text { monto de } \\
\text { ventas } \\
\text { anuales } \\
\text { (mdp) }\end{array}$ & $\begin{array}{l}\text { Tope máximo } \\
\text { combinado* }^{*}\end{array}$ \\
\hline Micro & Todas & Hasta 10 & Hasta $\$ 4$ & 4.6 \\
\hline \multirow[b]{2}{*}{ Pequeña } & Comercio & $\begin{array}{l}\text { Desde } 11 \\
\text { hasta } 30\end{array}$ & $\begin{array}{c}\text { Desde } \$ 4.01 \\
\text { hasta } \$ 100\end{array}$ & 93 \\
\hline & $\begin{array}{c}\text { Industria y } \\
\text { Servicios }\end{array}$ & $\begin{array}{l}\text { Desde } 11 \\
\text { hasta } 50\end{array}$ & $\begin{array}{c}\text { Desde } \$ 4.01 \\
\text { hasta } \$ 100\end{array}$ & 95 \\
\hline \multirow{3}{*}{ Mediana } & Comercio & $\begin{array}{l}\text { Desde } 31 \\
\text { hasta } 100\end{array}$ & \multirow{2}{*}{$\begin{array}{c}\text { Desde } \$ 100.01 \\
\text { hasta } \$ 250\end{array}$} & \multirow{2}{*}{235} \\
\hline & Servicios & $\begin{array}{l}\text { Desde } 51 \\
\text { hasta } 100\end{array}$ & & \\
\hline & Industria & $\begin{array}{l}\text { Desde } 51 \\
\text { hasta } 250\end{array}$ & $\begin{array}{c}\text { Desde } \$ 100.01 \\
\text { hasta } \$ 250\end{array}$ & 250 \\
\hline
\end{tabular}

*Tope Máximo Combinado = (Trabajadores) X 10\% + (Ventas Anuales) X 90\%.

Fuente: Diario Oficial de la Federación (2009).

En el mencionado acuerdo se indica que las micro, pequeñas y medianas empresas son un elemento fundamental para el desarrollo económico de los países, tanto por su contribución al empleo, como por su aportación al Producto Interno Bruto, constituyendo, en el caso de México, más del 99\% del total de las unidades económicas del país, representando alrededor del 52\% del Producto Interno Bruto y contribuyendo a generar más del 70\% de los empleos formales. Al precisar que estos datos se refieren a empleos formales, quiere decir que se trata de establecimientos identificables en cuanto a un domicilio, sin embargo no garantiza su cumplimiento con sus obligaciones en materia de prestaciones laborales, así como en materia de contribuciones fiscales, lo que abre una oportunidad para facilitar su integración a la formalidad.

\section{Planteamiento y delimitación del problema}

El presente trabajo de investigación busca resaltar el papel relevante que representan las mpymes en la economía mexicana, principalmente en la generación de empleos, así mismo se plantea como estrategia para desarrollar su competitividad el modelo canvas a través de una adaptación del mismo, atendiendo a las necesidades y características del entorno económico de Lázaro Cárdenas, Michoacán, de tal forma que contribuya al crecimiento económico de la región y del país. De lo anterior surge el siguiente interrogante: ¿La adaptación del modelo canvas servirá como herramienta competitiva a las mpymes de Lázaro Cárdenas, Michoacán? 


\section{A. Objetivo de la investigación}

Adaptar el modelo canvas como una herramienta de plan de negocios para las mpymes de Lázaro Cárdenas, Michoacán, que les permita desarrollar su competitividad.

\section{B. Supuestos de la investigación}

La problemática identificada, permite el establecimiento de los siguientes supuestos:

1) Las mpymes son más competitivas al adoptar el modelo canvas adaptado como herramienta de plan de negocios.

2) Si las mpymes no adoptan herramientas de plan de negocios como el método canvas adaptado, difícilmente prosperarán y permanecerán en el mercado.

\section{Justificación}

En los últimos años en México las mpymes, tomando en cuenta la gran cantidad de establecimientos que representan, han despertado el interés por parte de los diferentes niveles de gobierno, buscando establecer mecanismos de respaldo para su desarrollo, sin que a la fecha los resultados hayan solucionado del todo la problemática que enfrentan. Las micro, pequeñas y medianas empresas desempeñan un papel importante en el desarrollo económico de las naciones, tanto en los países industrializados, como en los de menor grado de desarrollo; como es el caso de México (Senado, 2002).

Según la Encuesta Nacional sobre Productividad y Competitividad de las Micro, Pequeñas y Medianas Empresas, presentada por el Instituto Nacional de Estadística y Geografía (INEGI), el Instituto Nacional del Emprendedor (INADEM) y el Banco Nacional de Comercio Exterior (BANCOMEXT), correspondiente a 2015, de acuerdo con los resultados el $97.6 \%$ de las empresas consideradas son microempresas y concentran el $75.4 \%$ del personal ocupado. Estas cifras reflejan la importancia de este sector económico y la necesidad de buscar su consolidación y crecimiento, como una respuesta a los bajos niveles de crecimiento de la economía nacional en los últimos años, así como una forma de fortalecer el empleo. La Tabla 3 muestra los números en materia de ocupación 
Tabla 3.

Número de empresas por tamaño y personal ocupado

\begin{tabular}{|l|c|c|c|}
\hline \multirow{2}{*}{ Tamaño } & \multicolumn{2}{|c|}{ Empresas } & Personal Ocupado \\
\cline { 2 - 4 } & Número & Participación (\%) & Participación (\%) \\
\hline Micro & 3.952 .422 & 97.6 & 75.4 \\
\hline Pequeña & 79.367 & 2.0 & 13.5 \\
\hline Mediana & 16.754 & 0.40 & 11.10 \\
\hline Total & 4.048 .543 & 100.0 & 100.0 \\
\hline
\end{tabular}

Fuente: Instituto Nacional del Emprendedor (2015).

Las microempresas desde el punto de vista numérico, revelan su destacada participación por la cantidad de establecimientos y un $75.4 \%$ del total de personal ocupado por las mpymes. Las pequeñas representan un $2 \%$ de este grupo y su contribución al empleo es del $13.5 \%$ del total, superando a las medianas que coadyuvan únicamente con el $11.10 \%$ en este rubro. Esta información fortalece la necesidad de atender estos establecimientos, mediante estrategias que aseguren su permanencia, crecimiento y competitividad, para influir de esta forma en los niveles de crecimiento interno, así mismo atender la demanda de trabajo de las nuevas generaciones en México.

El modelo canvas por su diseño es práctico y sencillo de aplicar, sin embargo el entorno de Lázaro Cárdenas y del país con características particulares, requiere de un amoldamiento que le sea aplicable a las mpymes de nueva creación y de aquellas ya establecidas, facilitando el desarrollo de un plan de negocios que guíe el rumbo de las mismas, para evitar la improvisación que pone en riesgo sus resultados a corto, mediano y largo plazo.

\section{Marco teórico conceptual}

\section{A. Modelo Canvas}

El modelo canvas creado por Alexander Osterwalder es una herramienta visual compuesta por un rectángulo dividido en nueve bloques que transforma la propuesta de servicios o productos en recursos económicos (De Oto, 2016).

El modelo canvas fue constituido con el fin de establecer una relación lógica entre cada uno de los componentes de la organización y todos los factores que influyen para que tenga o no éxito. A través de un "lienzo" se detalla desde la idea de negocios, hasta los diferentes factores que influirán en ella, al momento de ponerla en marcha (Ferreira D. C., 2015).

El modelo de negocio se divide en nueve módulos básicos que cubren las cuatro áreas principales: clientes, oferta, infraestructura y viabilidad. Estos nueve módulos son (Osterwalder, 2012): 
1. Segmentos de mercado: de masas, nicho de mercado, diversificado, multilateral. En este módulo se definen los diferentes grupos de personas o entidades a los que se dirige una empresa.

2. Propuestas de valor: novedad, mejora del rendimiento, personalización, "trabajo hecho", diseño, marca/estatus, precio, reducción de costes, reducción de riesgos, accesibilidad, comodidad/utilidad. En este módulo se describe el conjunto de productos y servicios que crean valor para un segmento de mercado específico.

3. Canales: directos, indirectos, propios y de socios comerciales. Se explica el modo en que una empresa se comunica con los diferentes segmentos de mercado para llegar a ellos y proporcionarles una propuesta de valor.

4. Relaciones con clientes: asistencia personal (exclusiva o no), autoservicio, servicios automáticos, comunidades, creación colectiva. Se describen los diferentes tipos de relaciones que establece una empresa con determinados segmentos de mercado.

5. Fuentes de ingresos: ventas de activos, cuota por uso, cuota de suscripción, préstamo/ alquiler/leasing, concesión de licencias, gastos de corretaje, publicidad. Se enfoca al flujo de caja que genera una empresa en los diferentes segmentos de mercado (para calcular los beneficios, es necesario restar los gastos de los ingresos).

6. Recursos clave: físicos, intelectuales, humanos y económicos. En este módulo se describen los activos para que un modelo de negocio funcione.

7. Actividades clave: producción, resolución de problemas, plataforma/red. Considera las acciones más importantes que debe emprender una empresa para que su modelo de negocio funcione.

8. Asociaciones clave: optimización y economía de escala, reducción de riesgos e incertidumbre, compra de determinados recursos y actividades. Describe la red de proveedores y socios que contribuyen al funcionamiento de un modelo de negocio.

9. Estructura de costos: costos fijos, costos variables, economías de escala, economías de campo. En este último módulo se describen todos los costes que implica la puesta en marcha de un modelo de negocio.

Los módulos anteriores propuestos por Osterwalder (2012), para la generación de modelos de negocio cubren cuatro áreas principales; clientes, oferta, infraestructura y viabilidad financiera. Para su mejor comprensión se presenta la Figura 1. 


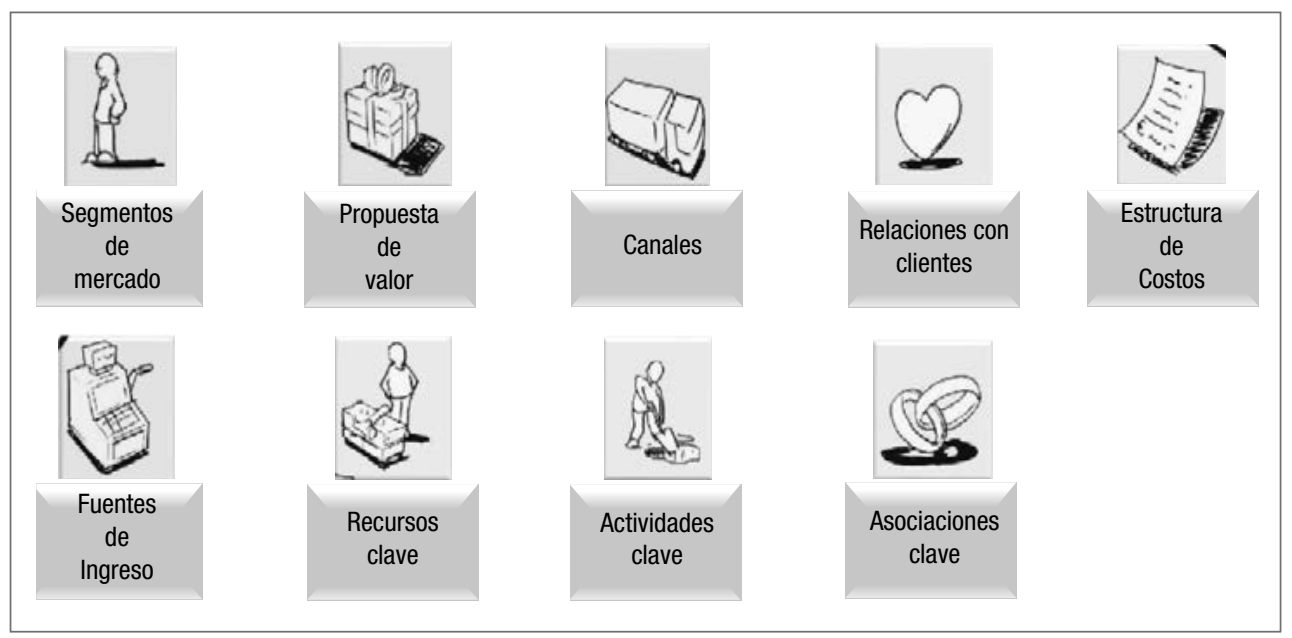

Figura 1. Los nueve módulos

Fuente: Osterwalder \& Pigneur (2011).

Los módulos anteriores se plasman en un lienzo, con la finalidad de que los participantes puedan comentar sobre el modelo de negocio. Esta propuesta resulta sumamente práctica, sin embargo, como ya se mencionó con anterioridad, es necesario adaptarla a las mpymes de Lázaro Cárdenas, Michoacán, considerando sus características particulares y el entorno en que se desarrollan. Un punto clave que no considera esta propuesta es el relativo a la inversión inicial, información necesaria para proyectos de nuevas empresas, así mismo es válido considerar la planeación estratégica a fin de identificar el rumbo de la organización. Otro punto a considerar es la determinación del costo unitario, por ser el punto de partida de un precio de venta competitivo, se menciona la estructura de los mismos, sin embargo no se precisa su integración.

\section{B. Plan de negocios}

Un plan de negocios "es un documento escrito de manera sencilla y precisa, el cual es el resultado de una planificación. Este documento muestra los objetivos que se quieren obtener y las actividades que se desarrollarán para lograr dichos objetivos” (Andía \& Paucara, 2013).

Es un resumen detallado sobre su empresa, los productos y servicios que suministra, las operaciones comerciales que desarrolla, cuáles son sus planes futuros, y el financiamiento con que cuenta y el que está requiriendo para su evolución empresarial (Banco de la Nación, 2013).

Un plan de negocios es una guía para el emprendedor o empresario. Se trata de un documento en el que se describe un negocio, se analiza la situación del mercado y se establecen las acciones que se realizarán en el futuro, junto con las correspondientes estrategias que serán implementadas, tanto para la promoción, como para la fabricación, si se tratara de un producto (Ferreira D. C., 2015). 


\section{Competitividad}

Competitividad se define como la calidad del ambiente económico e institucional para el desarrollo sostenible y sustentable de las actividades privadas y el aumento de la productividad; y a nivel empresa, la capacidad para mantener y fortalecer su rentabilidad y participación de las MIPYMES en los mercados, con base en las ventajas asociadas a sus productos o servicios, así como a las condiciones en que los ofrecen (Camara de Diputados, 2016).

La competitividad se define como el conjunto de instituciones, políticas y factores que determinan el nivel de productividad de un país. Dicho nivel, a su vez, establece el nivel de prosperidad que se puede ganar por una economía (Forum, The Global Competitiveness Report, 2014).

La competitividad es lo que permite a una empresa ser exitosa en el país en el que se desenvuelve, una empresa es competitiva cuando desarrolla servicios y productos cuyos costos de producción y calidad son comparables o superiores a las de sus competidores en todo el mundo. Se tiene que contar con un entorno físico, legal y regulatorio que contribuya a reducir los costos de producción y a elevar la productividad (Cervantes, Ballesteros, \& Hernández, 2012).

La competitividad puede analizarse desde dos perspectivas. En primer lugar, como un conjunto de factores que establecen el nivel de productividad y, segundo, como un determinante del incremento sostenido del bienestar de las personas. A partir de estas perspectivas, la competitividad regional puede ser definida como la administración de recursos y capacidades para incrementar sostenidamente la productividad empresarial y el bienestar de la población de la región (Benzanquen, Del Carpio, Zagarra, \& Valdivia, 2010).

\section{Micro, pequeñas y medianas empresas}

Las empresas son una fuente de ingresos para trabajadores, proveedores, gobierno y empresarios, promueven el desarrollo económico de la sociedad, contribuyen al sostenimiento de servicios públicos, representan un medio para la realización o satisfacción personal y fomentan la capacitación y el desarrollo de habilidades, así como la inversión y la formación de capital (Münch, 2006).

En el ámbito fiscal mexicano según el Código Fiscal de la Federación (2016), se considera empresa la persona física o moral que realice actividades comerciales, industriales, agrícolas, ganaderas, de pesca o silvícolas, ya sea directamente, a través de fideicomiso o por conducto de terceros.

De conformidad con la Ley para el Desarrollo y la Competitividad de la micro, pequeña y mediana empresa, en México se les define como micro, pequeñas y medianas empresas legalmente constituidas, de acuerdo con el número de trabajadores, con base en la estratificación establecida por la Secretaría de Economía, de común acuerdo con la secretaría de Hacienda y Crédito Público y publicada en el Diario Oficial de la Federación. (ver Tabla 4). 
Tabla 4.

Estratificación por número de trabajadores de la Secretaría de Economía, en común acuerdo con la Secretaría de Hacienda y Crédito Público

\begin{tabular}{|l|c|c|c|}
\hline \multicolumn{4}{|c|}{ Estratificación por número de trabajadores } \\
\hline Sector/Tamaño & Industria & Comercio & Servicios \\
\hline Micro & $0-10$ & $0-10$ & $0-10$ \\
\hline Pequeña & $11-50$ & $11-30$ & $11-50$ \\
\hline Mediana & $51-250$ & $31-100$ & $51-100$ \\
\hline
\end{tabular}

Fuente: Ley para el Desarrollo y la Competitividad de la micro, pequeña y mediana empresa (2016).

\section{Metodología}

Para el desarrollo de la presente investigación, la metodología empleada está basada en un enfoque empírico con análisis cualitativo, tomando como base fuentes electrónicas, literarias y científicas, así mismo información estadística gubernamental, obteniendo así la información considerada útil para el estudio, por su grado de aportación a la problemática a resolver. Se estima un estudio descriptivo pues "busca especificar las propiedades, las características y los perfiles de personas, grupos, comunidades, procesos, objetos o cualquier otro fenómeno que se someta a un análisis” (Hernández, Fernández, \& Pilar, 2014).

Se considera en el presente estudio como variable dependiente las mpymes de Lázaro Cárdenas, Michoacán y como independiente la adaptación del modelo canvas como una herramienta de plan de negocios que les permita desarrollar su competitividad.

\section{Análisis de resultados}

Tal y como se expresó con anterioridad, el modelo canvas es necesario adaptarlo a las mpymes de Lázaro Cárdenas, Michoacán, considerando sus características particulares y el entorno en que se desarrollan. Los módulos propuestos se plasman en un lienzo, con la finalidad de que los participantes puedan visualizar y comentar sobre el modelo de negocio.

Esta propuesta resulta sumamente práctica, sin embargo, como ya se mencionó con anterioridad, es necesario adaptarla a las mpymes de Lázaro Cárdenas, Michoacán, examinando sus características particulares y el entorno en que se desarrollan. Un punto importante que no avala esta propuesta es la determinación del costo unitario, por ser el punto de partida de un precio de venta competitivo, se menciona la estructura de los mismos en el modelo canvas, sin embargo, no se precisa su integración. Tampoco contempla datos relativos a la inversión inicial, información necesaria para proyectos de nuevas empresas., Así mismo es fundamental estimar la mercadotecnia básica, si bien es cierto en este modelo se considera pero de manera parcial. Finalmente la planeación estratégica a fin de identificar el rumbo que la organización ha de tomar. A continuación se describen los puntos propuestos. 


\section{A. Costo unitario}

Principalmente para empresas mpymes industriales, es importante integrar cada uno de los elementos que integran el costo por unidad producida, de igual manera las de servicios, para estar en posibilidad, una vez agregados los cargos por distribución, de establecer un precio de venta competitivo, que garantice un margen de utilidad apropiado. Para su determinación intervienen los siguientes elementos:

1) Materiales directos o materia prima. Lo integran los materiales o materia prima a transformar, se pueden cuantificar con facilidad e identificar físicamente en cada unidad producida. La materia prima es un elemento importante en la elaboración de un producto, ya que son todos los materiales susceptibles de transformación, los cuales darán origen a un producto elaborado diferente a los materiales conjugados y los cuales tendrán plena identificación con el artículo producido (Parra, 2009).

2) Mano de obra directa. Lo constituye los salarios y prestaciones que recibe el trabajador de producción, es decir; el que interviene de manera directa en la transformación de los materiales. La mano de obra representa el esfuerzo del trabajo humano que se aplica en la elaboración del producto. La mano de obra, así como la materia prima, se clasifica en mano de obra directa e indirecta. La mano de obra directa la constituye el esfuerzo laboral que aplican los trabajadores físicamente relacionados con el proceso productivo, sea por acción manual u operando una máquina (Sinisterra, 2011).

3) Gastos indirectos de fabricación. Lo integran conceptos como la mano de obra indirecta, la depreciación de la maquinaria y equipo, el mantenimiento, así mismo aquellos gastos que son de menor cuantía y que para su aplicación muchos de ellos se requiere prorratear. Además de la mano de obra y las materias primas, hay otros tipos de costos indirectos que son necesarios para fabricar el producto o proporcionar el servicio. Incluyen los costos de las instalaciones, el equipo utilizado para fabricar el producto o proporcionar el servicio y otro equipo de apoyo, como el que se emplea para el manejo de los materiales (Blocher, Stout, Gary, \& Chen, 2008).

Las empresas comerciales no tienen que integrar los conceptos anteriores, pues los productos que adquieren el precio de compra (este se conoce como costo de adquisición) es el mismo que se adicionará con los costos de distribución y el margen de utilidad, para así determinar el precio de venta. En la medida que estos conceptos sean conocidos se está en posibilidad de su optimización, lo que se traduce en mayor competitividad en el mercado.

\section{B. Inversión inicial}

Para iniciar una nueva empresa micro, pequeña o mediana es fundamental conocer además el capital necesario para su arranque, principalmente para definir el origen de dichos recursos, ya sea por aportación del o los propietarios, financiamiento por instituciones bancarias o gubernamentales, o en su caso una combinación de estos. Para destinarse fundamentalmente a: 
1) Permisos y gastos de constitución. En este apartado se consideran conceptos como los gastos notariales para la constitución, licencias, permisos y registros ante autoridades federales, estatales y locales.

2) Gastos preoperativos. Se deben considerar gastos a efectuar antes de iniciar operaciones, tales como contratación de servicios, depósito por renta de inmuebles, publicidad previa, sueldos, servicios de consultoría, entre otros.

3) Costo inicial de producción. Es la cuantificación de la producción inicial, en su caso el inventario necesario para arrancar operaciones, tratándose de negocios comerciales. Los establecimientos que transforman materia prima o productos semiterminados consideran además de estos conceptos, los sueldos directos e indirectos, pago de impuestos derivados de salarios, gastos tales como luz, mantenimiento de equipo, que necesitan ser erogados y que se ocupa conocer a efecto de contar con los recursos suficientes, hasta el momento en que comienza la recuperación de efectivo por la realización de ventas y servicios. Es decir, mantener en marcha la entidad hasta la generación de sus propios recursos.

4) Inversión fija. Se compone de los activos fijos necesarios para llevar a cabo la actividad principal, mismos que se adquieren con la intención de utilizarse tales como maquinaria de producción, terrenos, edificios, equipo de transporte, mobiliario, equipo de cómputo. etc.

Al contar con esta información, se está en posibilidad de cuantificar los recursos económicos necesarios para iniciar la nueva empresa, así como definir el origen de los recursos, ya sea por aportación del o los inversionistas, en su caso por financiamiento o la combinación de ambos.

\section{Mercadotecnia básica}

El producto o servicio que se desea colocar en el mercado debe2 ir precedido de estrategias que contribuyan en su éxito, si bien es cierto existen conceptos de décadas anteriores mismos que han sido modificados por los estudiosos del tema, sin embargo, siguen siendo el punto de partida en la comercialización, es el caso de las 4 P's o mezcla de la mercadotecnia, que en el modelo que se plantea como mercadotecnia básica, se adiciona con segmentos de mercado creado en un módulo por separado por Osterwalder \& Pigneur (2011), así como la identificación de la competencia que en los incisos siguientes se explica de manera sencilla, para facilitar su entendimiento y aplicación por las mpymes, como estrategia para su desarrollo. La Mezcla de Mercadotecnia es definida como un grupo de herramientas de marketing que son combinadas para producir la respuesta a la cual se quiere llegar con respecto al mercado meta (Kotler, 2010).

1) Producto. Producto es cualquier objeto o cosa susceptible de comprar o vender, sin embargo, existen aspectos importantes a considerar antes de su comercialización tales 
como el nombre, envase, presentación, frase publicitaria, logotipo y marca, entre otros, que deben planearse de tal forma que causen un impacto positivo en el consumidor y que contribuyan en la identificación del bien o servicio y la protección del mismo. Además de cerciorase en el uso de nombre, registrarlo, en su caso para evitar problemas legales por utilizar uno ya existente en el mercado.

2) Precio. Para el consumidor la parte monetaria es importante, vinculándola con la calidad requerida y desde luego comparándola con el que ofrece la competencia, de tal manera que debe ser competitivo y que además incluya el margen de utilidad que pretende el empresario, en su caso, es punto de partida en la búsqueda de alternativas para la optimización de costos, sin embargo, en este apartado las mpymes deben definir también la forma de pago, ya sea transferencia bancaria, depósito en cuenta, efectivo o tarjeta de crédito. Actualmente el dinero electrónico es utilizado de manera generalizada en México, en parte apoyado por las disposiciones fiscales vigentes, que obligan a utilizar estos medios al cubrir adquisiciones y gastos que se pretende deducir de impuestos.

3) Plaza. En este apartado, el empresario debe analizar y decidir la manera en que hará llegar al consumidor sus productos, es decir; el canal de distribución adecuado de acuerdo con las características propias, buscando que sea en el tiempo y la forma correctos, sin que este proceso impacte de manera significativa el precio de venta. Puede ser tiendas, venta directa, por internet, distribuidores etc. La ubicación y la facilidad para acceder al establecimiento son factores claves a tomar en cuenta para mejores resultados.

4) Promoción. Para todo negocio es primordial identificar el o los medios para darse a conocer, principalmente con el mercado al que va dirigido, considerando los diferentes hábitos de los consumidores para consultar información antes de efectuar la compra, tales como Internet, radio, periódico, exposición en ferias, promoción personalizada etc. Kotler, 2010, atinadamente afirma que las compañías deben contactarse con los clientes actuales y potenciales, sin dejar al azar lo que desean comunicar. Lo importante es difundir lo que se quiere dar a conocer, para lograr que el consumidor quede convencido y tome una decisión que sea favorable.

5) Segmentos de mercado. Como lo plantea Osterwalder \& Pigneur (2011), l as empresas deben seleccionar, con una decisión fundamentada, los segmentos a los que se van a dirigir y, al mismo tiempo, los que no tendrán en cuenta. Una vez que se ha tomado esta decisión, ya se puede diseñar un modelo de negocio basado en un conocimiento exhaustivo de las necesidades específicas del cliente objetivo. Definitivamente es determinante que las mpymes identifiquen quiénes son sus clientes potenciales, adónde dirigen su producto o servicio, así como la capacidad de la demanda del mercado meta. Esta información aportará datos relevantes sobre su factibilidad de mercado.

6) Identificación de la competencia. Así como es importante conocer el mercado al que va dirigido el producto o servicio, es trascendental enterarse quiénes y cuántos son 
los competidores, cuáles son sus fortalezas, qué están ofreciendo para mejorarlos, la calidad de sus bienes y servicios. Las mpymes al tener conocimiento de ello entienden con claridad lo que necesitan para lograr competir y penetrar en el mercado, sin que su incursión sea resultado de la improvisación.

En los nueve módulos propuestos por Osterwalder \& Pigneur (2011), en el relativo a la propuesta de valor se abordan puntos como precio, diseño y marca. Así mismo en el referente a los canales se exponen los aspectos concernientes a la distribución y venta, sin embargo, por cuestionar prácticas es más sencillo conjuntar en un solo apartado, como se presenta en la mercadotecnia básica que se propone tomando en consideración que las mpymes en México están dirigidas por un miembro de la familia o en todo caso no cuentan con una estructura administrativa que ofrezca conocimientos multidisciplinarios, siendo necesario facilitar su asimilación.

\section{Planeación estratégica}

Toda organización necesita saber su razón de ser, hacia dónde dirigirse para lograr sus objetivos, conocer internamente sus capacidades y la situación que prevalece en el mercado, qué tan propicio es o en su defecto las turbulencias que se presentan. Básicamente las mpymes requieren definir en este apartado lo siguiente:

1) Misión. Toda empresa cuenta con una actividad a desarrollar, que se establece como parte integral de su documento constitutivo o registro ante diferentes organismos gubernamentales, pero más allá de la actividad es entender la razón de ser de la misma, el motivo de su existencia y que cada miembro la conozca y se identifique con ella, para que se convierta en un propósito de la organización. "La misión es la razón de ser de la organización, su formulación es una etapa de las más importantes en el proceso de planeación, es una definición amplia del propósito de la organización y la descripción del negocio al que se dedica la compañía” (Munch, 2013).

2) Visión. Toda organización debe vislumbrar su futuro, cómo se ve en el corto, mediano y largo plazo. La visión corresponde al futuro deseado de la organización. Munch (2013), la define como el enunciado que describe el estado deseado en el futuro. Provee dirección y forja el futuro de la organización estimulando acciones concretas en el presente.

Es necesario que los objetivos organizacionales a corto, mediano y largo plazo queden plasmados de manera específica, para permitir su control respecto a su ejecución y resultados. Esto facilitará a las mpymes asegurarse del rumbo que deben tomar y establecer las medidas preventivas o correctivas en el momento oportuno, considerando los diversos escenarios que se van presentando a lo largo de su ciclo de vida.

3) Valores. Los valores que se inculcan en las organizaciones son los gobernadores de las acciones de sus miembros, representan la fuente de la cultura organizacional. Definen 
el conjunto de principios, creencias, reglas que regulan la gestión de la organización. Constituyen la filosofía institucional y el soporte de la cultura organizacional. El objetivo básico de la definición de valores corporativos es el de tener un marco de referencia que inspire y regule la vida de la organización y por ende lo que se está pidiendo tanto de los empleados de la misma, como en cuanto a la atención al cliente (Morales, 2014).

4) Análisis FODA. Es una herramienta para que las mpymes puedan analizar internamente sus cualidades y de igual manera su fragilidad como organización, y por supuesto es una manera también de explorar el entorno para visualizar su pertinencia y los desafíos a enfrentar. Este tipo de empresas cuenta con grandes fortalezas, que finalmente definen su éxito, por tanto es importante conocerlas para explotarlas al máximo, atendiendo las debilidades que en su caso constituyan un obstáculo en el logro de sus objetivos.

El análisis FODA es un instrumento de diagnóstico utilizado por una empresa u organización con la finalidad de intervenir profesionalmente tanto en la formulación y aplicación de estrategias como en su seguimiento para hacer una evaluación y control de resultados. Consiste en realizar una evaluación de los factores fuertes y débiles, que diagnostican la situación interna de una organización, así como la evaluación externa, marcada por las oportunidades y las amenazas (Valda, 2012).

5) Mejora continua. Es un concepto del siglo anterior, sin embargo las organizaciones actualmente se enfrentan a una competencia muy concurrida en los mercados, los cambios se dan de manera vertiginosa, situación que obliga a las mpymes a establecer una filosofía centrada en aumentar la calidad del producto o servicio, así como optimizar los procesos a fin de que satisfagan las necesidades y requerimientos de los clientes, logrando con ello conservarlos y atraer otros nuevos. Con el análisis FODA se identifican los puntos débiles para establecer soluciones, mediante estrategias tales como capacitación, revisión de los procesos, controles etc., para elevar la productividad.

Es fundamental establecer un sistema de gestión para la calidad claramente orientado a los procesos y a la mejora continua. Las organizaciones lograrán el liderazgo en la medida que tengan la habilidad para mantener la excelencia de sus procesos y se comprometan con el constante desarrollo de sus objetivos, siempre orientadas a la satisfacción de sus clientes (García, Quispe, \& Páes, 2003).

El modelo propuesto queda compuesto por ocho módulos, incluyendo el de costo unitario, inversión inicial, mercadotecnia básica y planeación estratégica, conservando de los establecidos por Osterwalder \& Pigneur (2011 el de fuente de ingresos, propuesta de valor, relación con los clientes y asociaciones clave, quedando como a continuación se observa (Figura 2). 


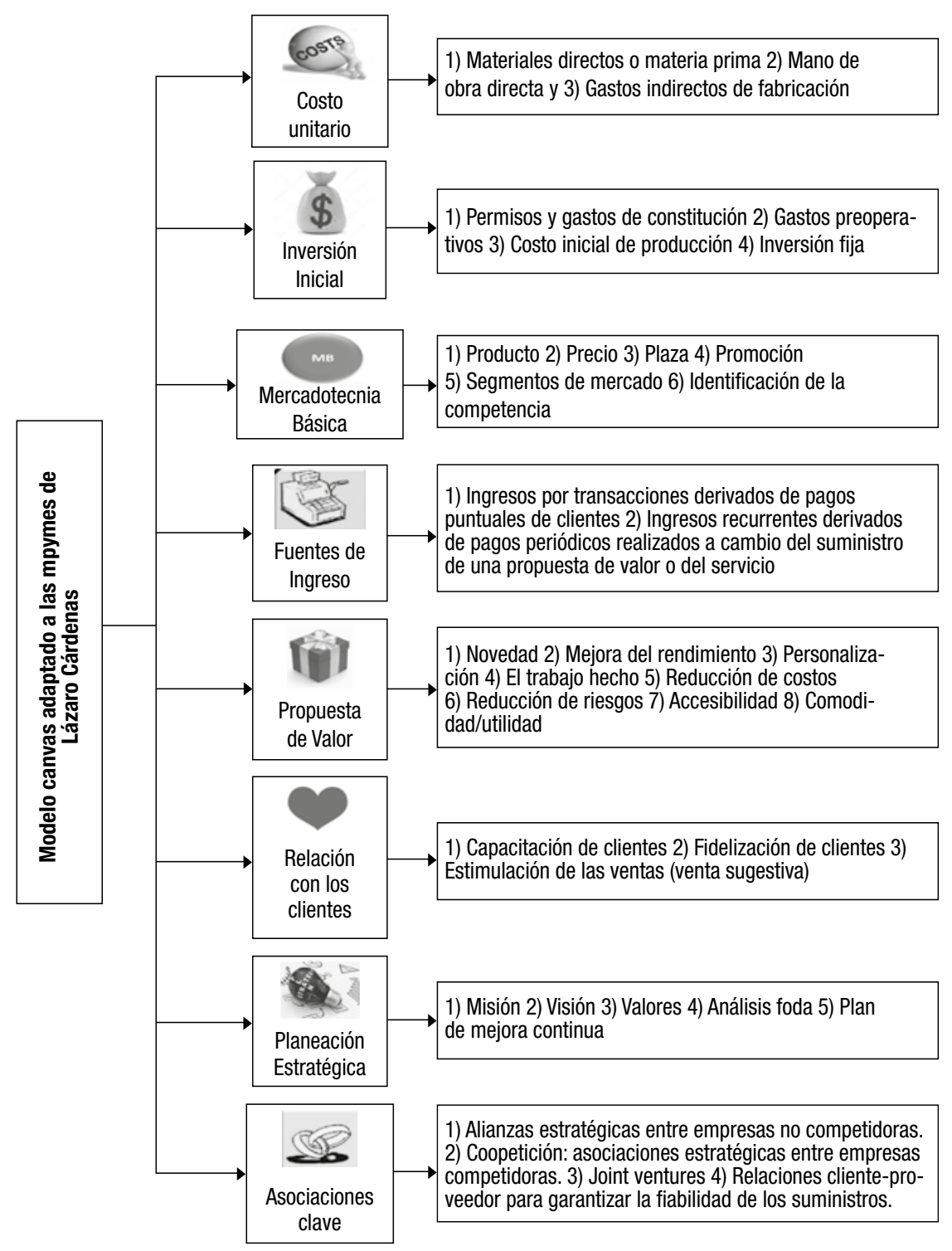

Figura 2. Modelo de negocios canvas analítico, adaptado a las mpymes de Lázaro Cárdenas

Fuente: Elaboración propia, basada en el modelo canvas de Osterwalder \& Pigneur (2011).

En la figura anterior, comparándola con la propuesta por Osterwalder \& Pigneur (2011), se suprimierón los siguientes módulos:

1) Actividades clave

2) Estructura de costos 

3) Recursos clave
4) Segmentos de mercado.
5) Canales

Las tareas correspondientes a ellos quedaron contempladas en los apartados que se adicionaron, de manera más específica, facilitando su aplicación y entendimiento, principalmente considerando las características de quienes dirigen las mpymes de Lázaro Cárdenas, Michoacán; a continuación se menciona los módulos que se adicionaron:
1) Costo unitario
2) Inversión inicial
3) Mercadotecnia básica
4) Planeación estratégica

El modelo de negocios propuesto guarda un orden de acuerdo con las necesidades de información que se van ocupando para su aplicación adecuada, de igual manera puede plasmarse en un lienzo para facilitar su desarrollo, como se presenta en la Figura 3, conteniendo los datos principales, para en cada módulo desagregar la información específica y mencionada y resumida en la Figura 2.

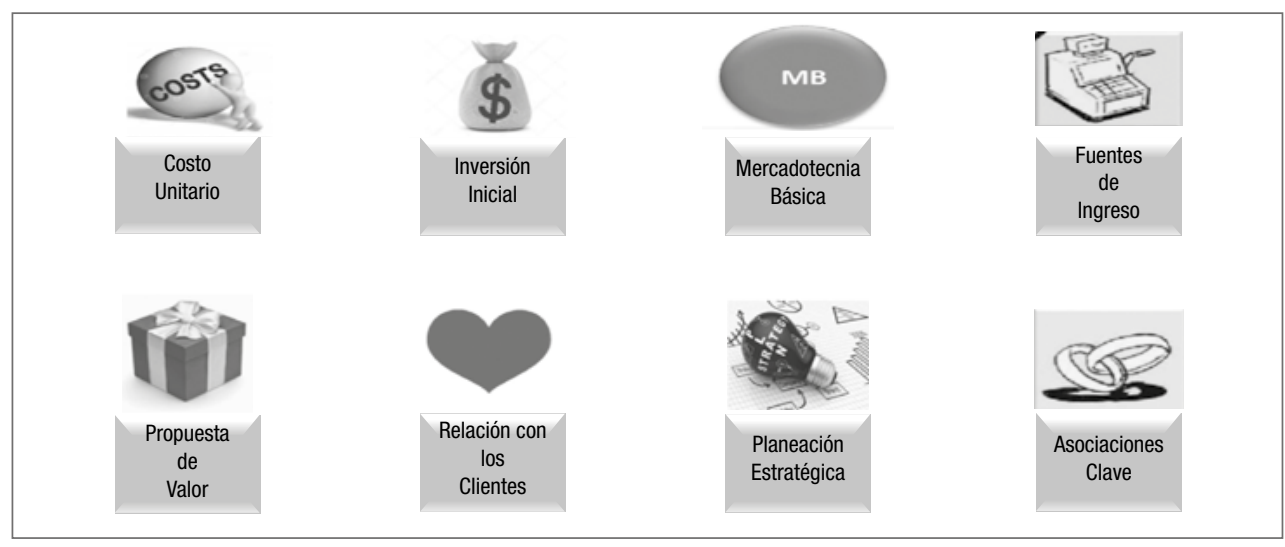

Figura 3. Modelo de negocios canvas adaptado a las mpymes de Lázaro Cárdenas

Fuente: Elaboración propia, basada en el modelo canvas de Osterwalder \& Pigneur (2011).

\section{Conclusiones y recomendaciones}

Las mpymes, por su contribución a la economía de México, representan un factor importante de desarrollo, principalmente en la actualidad por los diferentes retos a enfrentar en cuanto a renegociación de tratados internacionales en materia comercial con Estados Unidos y Canadá, así como por las políticas migratorias en marcha con el país del norte. Por lo anterior, estas empresas requieren además de estímulos para asegurar su desarrollo, estrategias que les permita conocer su modelo de negocios de manera sistémica, visualizando los principales procesos que lo conforman a efecto de analizarlos, entenderlos y dirigir sus esfuerzos para lograr sus objetivos dentro de un ambiente de certidumbre. 
Para ello, la presente investigación concluyó con la adaptación del modelo canvas a las mpymes de Lázaro Cárdenas, Michoacán, como una estrategia para generar su competitividad, considerando un desarrollo simple para su mejor entendimiento, tomando en cuenta que estas empresas se encuentran dirigidas por una sola persona que realiza diferentes actividades y por lo regular es miembro de la familia. Es decir, no cuentan con una estructura organizacional que garantice actividades multidisciplinarias especializadas; sin embargo mediante la propuesta que se presenta, de manera sencilla pueden abordarse los puntos estratégicos que desarrollan las actividades vitales de la organización plasmadas en un lienzo. Con lo anterior se contribuye al desarrollo de su competitividad.

El modelo canvas adaptado a las mpymes de Lázaro Cárdenas, puede ser utilizado en cualquier organización, incluso ser modificado de acuerdo con las características de cada entorno, en su caso a la realidad económica, política y social que prevalezca, pues las economías no son estáticas, los cambios se siguen dando, de igual manera las propuestas son susceptibles de cambios y adaptaciones. En cuanto a los resultados, se recomienda que estos establecimientos, principalmente las microempresas, se integren a la formalidad como parte de la base de contribuyentes, simplificando su incorporación, a fin de que los beneficios se reflejen en la empleabilidad respecto a las prestaciones que se derivan y en la recaudación de impuestos de una manera justa y equitativa, sin que ello represente cargas adicionales o procesos de cumplimiento complejos.

\section{Declaración conflicto de intereses}

Los autores declaran no tener ningún conflicto de intereses

\section{Referencias bibliográficas}

1. Andía, W., \& Paucara, E. (2013). Los planes de negocios y los proyectos de inversión: similitudes y diferencias. Industrial Data, 80-84.

2. Banco de la Nación, A. (Marzo de 2013). Guía para empresarios PyMEs para elaborar un Plan de Negocios. Obtenido de Plan PYMES: http://rincondelemprendedor.es

3. Benzanquen, J., Del Carpio, L., Zagarra, L., \& Valdivia, C. (2010). Un Índice Regional de Competitividad para un país. CEPAL, 69-86.

4. Blocher, E. J., Stout, D. E., Gary, C., \& Chen, K. H. (2008). Administración de costos. México: McGraw-Hill .

5. Camara de Diputados, D. H. (Mayo de 2017). Ley para el desarrollo de la competitividad de la micro, pequeña y mediana empresa. México, México: Congreso de la Unión.

6. Cámara de Diputados, H. Congreso de la Unión (2016). Código Fiscal de la Federación. Recuperado de: www.diputados.gob.mx

7. Cervantes, M., Ballesteros, B., \& Hernández, F. (2012). Programas para elevar la competitividad de las empresas en México. Observatorio de la Economía Latinoamericana.

8. De Oto, G. B. (9 de Noviembre de 2016). Herramienta sencilla para aplicar en tu negocio. Obtenido de buenosnegocios.com: www.buenosnegocios.com 
9. Ferreira, D. C. (2015). El modelo canvas en la formulación de proyectos. Cooperativismo y desarrollo, 69-80.

10. Forbes, M. (13 de Mayo de 2016). Forbes Staff Economía y Finanzas. Obtenido de https://www.forbes.com.mx/

11. Forum, W. E. (2014). The Global Competitiveness Report. Ginebra: World Economic Forum.

12. García, M., Quispe, C., \& Páes, L. (2003). Mejora continua de la calidad en los procesos. Industrial Data, 89-94.

13. Góngora, J. (2013). El panorama de las micro, pequeñas y medianas empresas en México. Comercio exterior, 2-6.

14. Hernández, R., Fernández, C., \& Pilar, B. M. (2014). Metodología de la investigación. México: McGraw-Hill.

15. INEGI, Instituto Nacional del Emprendedor \& Banco, N. M. (2015). Encuesta Nacional sobre Productividad y Competitividad de las Micro, Pequeñas y Medianas Empresas. Obtenido de http://www.inegi.org.mx/saladeprensa/boletines/2016/especiales/ especiales2016_07_02.pdf

16. Kotler, P. (1 de Diciembre de 2010). Philip Kptler. Obtenido de http://phlpktler. blogspot.mx/

17. Morales, M. (1 de Agosto de 2014). pymempresario. Obtenido de http://www. pymempresario.com/2014/08/como-construir-una-filosofia-empresarial/

18. Münch, L. (2006). Fundamentos de Administración: Casos y prácticas. México: Trillas.

19. Munch, l. (2013). Planeación estratégica, el rumbo hacia el éxito. México: Trillas.

20. Osterwalder, A. (21 de Febrero de 2012). BBVA INNOVATION CENTER. Obtenido de Generación de modelos de negocio: http://www.centrodeinnovacionbbva.com

21. Parra, J. A. (2009). Costos Históricos. Agua Prieta : Instituto Tecnológico de Agua Prieta.

22. Senado, I. d. (Julio de 2002). Senado de la República. Obtenido de http://bibliodigitalibd. senado.gob.mx/handle/123456789/1718

23. Sinisterra, G. (2011). Contabilidad de costos. Bogotá: Litoperla Impresores.

24. Valda, J. C. (11 de Octubre de 2012). Grandes Pymes. Obtenido de http://www. grandespymes.com.ar/2012/10/11/analisis-f-o-d-a/ 